\title{
Les rivières fantômes du désert oriental
}

La topographie du Proche-Orient antique, et en particulier de la Mésopotamie avec les pays adjacents, repose largement sur les informations de Ptolémée ( $\left.{ }^{1}\right)$. Cependant, un grand nombre des noms géographiques relatifs à cette région qu'il nous a transmis restent difficiles, voire impossibles, à identifier sur le terrain. Ce n'est pas tant la faute de Ptolémée lui-même, ou de l'état du texte reçu, que le résultat de la pauvreté des données littéraires ou épigraphiques qui auraient pu recouper son témoignage. S'il est clair que les longues listes où la monotonie des chiffres va de pair avec l'étrangeté des noms favorisent des fautes de copie, comme l'apparat critique de Müller en témoigne, s'il est non moins sûr que les moyens de mesure antiques étaient très insuffisants, les données transmises forment malgré tout un ensemble cohérent avec une marge d'erreur raisonnable, chaque fois qu'il est possible de les vérifier. Malheureusement, ce n'est pas souvent le cas.

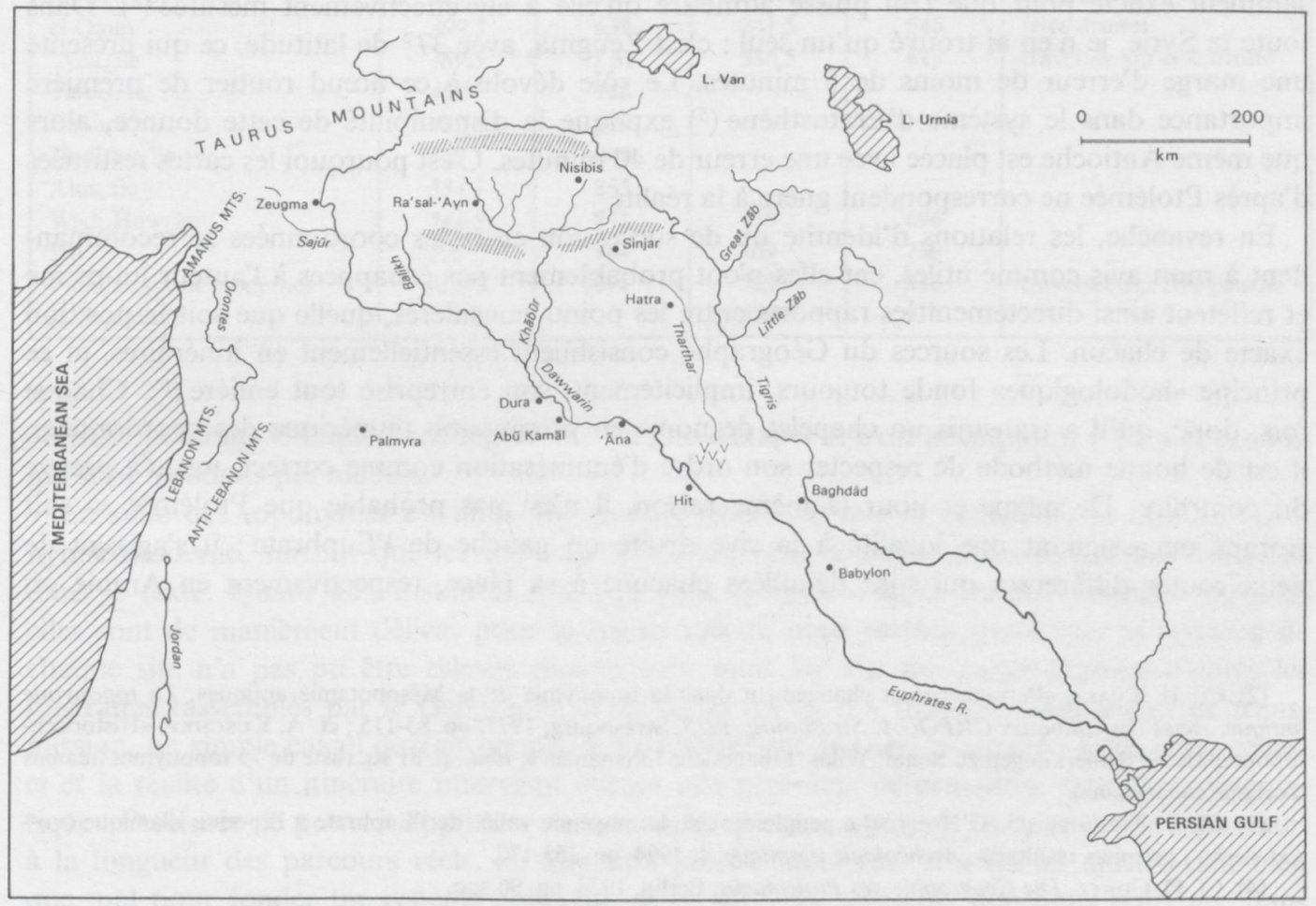

(1) Geogr., V, 14 (Syria), 17 (Mesopotamia), 18 (Arabia Deserta). J'utilise l'édition de C. Muller (Paris, 1901) toujours irremplaçable. 
La tentation est grande, dans ces circonstances, de suppléer cette déficience en utilisant la toponymie moderne. Bien entendu, cette méthode, utilisée avec modération, peut porter de bons fruits : les noms d'une grande antiquité se révèlent parfois d'une façon manifeste dans une appellation encore en usage de nos jours $\left({ }^{2}\right)$. Mais les richesses de la langue arabe (et parfois la fantaisie des informateurs) refusent rarement quelque prise à qui se contente des apparences. Le danger est particulièrement aigu dans les régions qui ont connu une longue interruption de la vie sédentaire. La vallée du Moyen-Euphrate en est une : encore au début du siècle dernier, il n'y avait pratiquement pas de villages entre Jerablûs et 'Ana. Il va de soi que, dans de telles conditions, la toponymie du pays tend à se renouveler $\left({ }^{3}\right)$.

Certains voyageurs s'en donnaient vraiment à cœur joie pour chercher (et trouver) des appellations modernes qui correspondissent plus ou moins bien à tel ou tel nom chez Strabon, chez Isidore, chez Ptolémée. Sans s'attarder sur les exemples (qui sont nombreux), il suffit de dire que même le grand René Dussaud a occasionnellement donné dans le piège. En même temps, on est surpris de constater le peu de cas que l'on faisait généralement des données numériques de Ptolémée dans ces comparaisons.

Il est vrai qu'il ne suffit pas de les transmettre sur le papier que l'on surimposerait ensuite sur un fond de carte moderne. En effet, sans parler des longitudes, que les Anciens ne pouvaient estimer que d'après les distances, rarement parcourues en ligne droite et dans le sens est-ouest, mêmes les latitudes n'ont pas été mesurées souvent, comme cela était théoriquement possible. Dans l'ouvrage entier de Ptolémée, rien que de rares points-clés donnent une latitude suffisamment exacte pour que l'on puisse admettre qu'elle a été effectivement mesurée $\left({ }^{4}\right)$. Dans toute la Syrie, je n'en ai trouvé qu'un seul : c'est Zeugma, avec $37^{\circ}$ de latitude, ce qui présente une marge d'erreur de moins de 5 minutes. Le rôle dévolu à ce nœud routier de première importance dans le système d'Ératosthène ${ }^{(5)}$ explique la disponibilité de cette donnée, alors que même Antioche est placée avec une erreur de 40 minutes. C'est pourquoi les cartes restituées d'après Ptolémée ne correspondent guère à la réalité.

En revanche, les relations d'identité ou de succession entre les coordonnées se recommandent à mon avis comme utiles, car elles n'ont probablement pas échappées à l'auteur lui-même et reflètent ainsi directement les rapports entre les points considérés, quelle que soit la position exacte de chacun. Les sources du Géographe consistaient essentiellement en itinéraires, et ce principe "hodologique» fonde toujours, implicitement, son entreprise tout entière $\left(^{6}\right)$. Chaque fois, donc, qu'il a transmis un chapelet de noms en progression numérique des coordonnées, il est de bonne méthode de respecter son ordre d'énumération comme correct, jusqu'à preuve du contraire. De même et pour la même raison, il n'est pas probable que Ptolémée se soit trompé en assignant une localité à la rive droite ou gauche de l'Euphrate; il s'agissait de deux routes différentes qui sont détaillées chacune à sa place, respectivement en Arabie ou

(2) Cf. H. Limet, «Permanence et changement dans la toponymie de la Mésopotamie antique», La toponymie antique, Actes des colloques CRPOGA, Strasbourg, 1975, Strasbourg, 1977, pp. 83-115 ; cf. A. KusCHKE, «Historischtopographische Bemerkungen zu Stefan Wilds 'Libanesiche Ortsnamen'», ibid., p. 81 sq. (liste de 75 toponymes libanais d'origine cananéenne).

(3) Cf. S. Berthier, O. D'Hont, «Le peuplement de la moyenne vallée de l'Euphrate à l'époque islamique (vire$\mathrm{xx}^{\mathrm{e}}$ siècle) : premiers résultats", Archéologie islamique, 4, 1994, pp. 153-175.

(4) Cf. O. Cuntz, Die Geographie des Ptolemaeus, Berlin, 1923, pp. 96 sqq.

(5) Comme je le montre ailleurs, Zeugma est identique à Thapsaque, un pivot du système d'Ératosthène (Cf. Strabo, Geogr., II, 1, 23-26).

(6) Sur cette vue de la géographie antique, cf. P. JANNI, La mappa e il periplo : cartografia antica e spazio odologico, Rome, 1984. 
TABLE I

La route de l'Euphrate (d'après Handbook of Mesopotamia, 1917)

\begin{tabular}{|c|c|c|c|c|c|}
\hline \multirow[b]{2}{*}{ De Zeugma (Belkis) à : } & \multicolumn{2}{|c|}{ sur eau } & \multicolumn{2}{|c|}{ sur terre (rive gauche) } & \\
\hline & milles & km & milles & $\mathbf{k m}$ & \\
\hline Birecik & 7,5 & 12 & & & \\
\hline Tell Ahmar (rive gauche) & 35,5 & 57 & 40 & 64 & \\
\hline Meskeneh & 97,5 & 156 & 142,5 & 228 & (rive droite) \\
\hline Dibsi & 111,5 & 178 & & & \\
\hline Q. Jaber (rive gauche) & 126,5 & 202 & & & \\
\hline Suriyeh & 145,5 & 233 & & & \\
\hline Tell Thadein & & & 146,5 & 234 & (rive droite) \\
\hline Hiraklia (rive gauche) & 161,5 & 258 & 155,5 & 249 & \\
\hline Raqqa (rive gauche) & 165,5 & 265 & 162,5 & 260 & \\
\hline Balikh, delta (rive gauche) & $166,5-173,5$ & $266-278$ & 170,5 & 273 & \\
\hline Halebiyeh & 231,5 & 370 & & & \\
\hline Deir ez-Zor & 278,5 & 445,5 & & & \\
\hline Buseira (rive gauche) & & & & & \\
\hline Embouchure du Khabûr & 309 & 494,5 & 274,5 & 439 & \\
\hline Doura-Europos & 348,5 & 557 & 302 & 483 & (rive droite) \\
\hline Abû Kemâl / Wadi Swâb & 382.5 & 612 & 325 & 520 & (rive droite) \\
\hline 'Irși (rive gauche) & 390,5 & 625 & 326,5 & 522 & \\
\hline Anqa & 395,5 & 633 & 337,5 & 540 & (rive droite) \\
\hline el-Qaim & 399,5 & 639 & 341,5 & 546 & (rive droite) \\
\hline 'Ana, île & 469,5 & 751 & 386,5 & 618 & traversée sur rive droite \\
\hline Talbis, île & 480 & 768 & & & \\
\hline Fahimi & 487 & 779 & & & \\
\hline Haditha, île & 525 & 840 & & & \\
\hline Alus, île & 534,5 & 855 & & & \\
\hline Wadi Hawrân & 544,5 & 871 & 435 & 696 & \\
\hline Hit & 590 & 944 & 469 & 750 & \\
\hline $\begin{array}{l}\text { Fallujah (pont moderne) } \\
\text { (à Baghdad) }\end{array}$ & 669.5 & 1071 & $\begin{array}{l}529 \\
572.5\end{array}$ & $\begin{array}{l}846 \\
916\end{array}$ & traversée sur rive gauche \\
\hline
\end{tabular}

en Mésopotamie. Chaque nom représente un gîte d'étape, et c'est pourquoi il y en a beaucoup qui sont inconnus par ailleurs.

La suite des toponymes est ainsi une donnée plus sûre que la comparaison avec la toponymie moderne, surtout que les noms de Ptolémée sont souvent et manifestement déformés dans le texte. Quant aux distances que l'on peut calculer d'après les coordonnées fournies, elles sont de maniement délicat pour la même raison, mais surtout parce que la position de chaque site n'a pas pu être relevée directement, mais lue sur des cartes établies d'après les itinéraires rassemblés par le Géographe et les distances qu'il y trouvait. Sauf erreur de transmission, un simple calcul devrait permettre de rétablir ces données premières, mais entre celles. ci et la réalité d'un itinéraire intervient encore une profonde déformation, cette fois systématique : Ptolémée ne pouvait appliquer sur ses cartes que des segments en ligne droite répondant à la longueur des parcours réels, de direction précise inconnue. Il a dû les ajuster tant bien que mal pour fonder un système cohérent, et ce sont ces segments que nous pouvons, dans le meilleur des cas, récupérer.

Autant le cours des grands fleuves mésopotamiens, l'Euphrate et le Tigre, qui servaient d'axes majeurs de communication, est ponctué chez Ptolémée de nombreux gîtes d'étape, autant 
d'autres cours d'eau reçoivent de sa part très peu d'attention, sans doute parce qu'ils ne figuraient pas dans les itinéraires utilisés, et donnent parfois lieu à des erreurs grossières. Il suffit de mentionner une rivière anonyme mais importante qu'il fait passer par Palmyre du nord-ouest au sud-est ; d'après les coordonnées, elle devait dépasser $40 \mathrm{~km}$ de longueur ( $\left.{ }^{7}\right)$. Je n'ai pas besoin de dire qu'il n'y a jamais eu en réalité rien de pareil, et le maigre ruisseau issu de la source Efqa n'a jamais pu avoir plus de $2 \mathrm{~km}$ de long. Même les aqueducs souterrains qui rejoignent Palmyre, à supposer pour un moment qu'ils aient pu par malentendu passer pour un seul cours d'eau naturel, viennent l'un par l'ouest et l'autre par le nord.

Personne, à ma connaissance, ne s'est attardé à cette absurdité géographique. Elle demeure inexplicable. En revanche, une autre rivière fantomatique a beaucoup sollicité l'ingéniosité des érudits. Je pense à Saokoras, que Ptolémée est seul à nommer et qu'il fait se jeter du côté est dans l'Euphrate en aval du Chaboras, nom où l'on reconnaît sans peine le Khabûr ${ }^{8}$ ).

Cependant, l'Euphrate ne reçoit de ce côté que le Balikh, bien en amont du Khabûr, et que Ptolémée passe sous silence. À part ces deux cours d'eau, on ne trouve sur la rive gauche du grand fleuve que des wadi toujours à sec sauf quelques jours par an. L'un et l'autre ont toujours eu une importance économique considérable, tant pour l'agriculture que pour les communications. L'un et l'autre aussi ont porté les mêmes noms depuis au moins le troisième millénaire jusqu'à nos jours et sont bien attestés sous ces mêmes noms, juste pourvus de désinences, dans les sources grecques et latines $\left({ }^{9}\right)$. La continuité onomastique est ici entière.

Une solution du problème a été avancée dès 1779 par le chevalier d'Anville : il a pensé pour Saokoras au Wadi Sûr, débouchant un peu en aval de 'Ana. Acceptée, faute d'information suffisante, par le géographe allemand Ritter qui a rassemblé une somme des connaissances géographiques accessibles dans les années quarante du xix siècle $\left({ }^{10}\right)$, cette possibilité n'a pas paru s'imposer au docteur Ainsworth, bien qu'il ait été amené, ayant momentanément perdu contact avec son bateau en 1836, à traverser ce wadi en eau par un pont (11). Kiepert n'était pas convaincu non plus, et il a omis le Saokoras de ses cartes. Il y a mis toutefois, influencé par les récits des voyageurs, le Wadi Sûr que la cartographie récente ne s'embarrasse plus d'indiquer. J'avoue que j'ai failli moi-même remarquer l'existence même du Wadi Sûr lorsque je prospectais, en 1979, le site assyrien de Sûr en face de l'île de Telbis; il est vrai que j'étais alors inconscient du problème qui m'occupe ici, mais le moins que l'on puisse en dire, c'est que ce prétendu affluent de l'Euphrate ne saute pas aux yeux.

La plupart des commentateurs ont pensé que Ptolémée avait, par quelque malentendu, appelé le Balikh Chaboras et le Khabûr Saokoras. Consacrée par la Realencyclopädie, cette explication paraît tout à fait plausible de prime abord, car la distance entre les confluents des deux rivières, calculée d'après leurs cordonnées comme établies dans l'édition de Müller, s'élève à $180 \mathrm{~km}$ environ ( $\left.{ }^{12}\right)$, à peu près autant que les $166 \mathrm{~km}$ entre les estuaires du Balikh et du Khabûr. Cette coïncidence ne résout cependant pas toutes les difficultés, car Ptolémée connaît aussi une localité du nom de Chabora qu'il place bien en aval de la ville de Nicéphorion

(7) Prol., V, 14, 7.

(8) Ptol., V, 13, 3.

(9) Cf. RE, I (1893), 107 (Fraenkel) ; H. Limet, op. cit., pp. 102 sqq.

(10) C. Ritter, Die Erdkunde... VII, Berlin, 1844, pp. 254 et 728.

(11) W. F. Ainsworth, A Personal Narrative of the Euphrates Expedition, London, 1888, I, p. 339 et II, p. 432 sq.

(12) Je compte avec H. I. McAdam, dans P.-L. Gatier, B. Helly, J.-P. Rey-Coquais (éd.), Géographie historique du Proche-Orient, p. 63 sq., $91 \mathrm{~km}$ pour $1^{\circ}$ de latitude et $73 \mathrm{~km}$ pour $1^{\circ}$ de longitude (parallèle de Rhodes), respectivement 500 et 400 stades (PTOL., I, 2 et VI I, 5). 
(qui était, comme on le sait par ailleurs, située précisément au confluent du Balikh, près de la moderne Raqqa), mais à une distance qui n'est que de quelque $70 \mathrm{~km}$ d'après les coordonnées, soit une bonne centaine de $\mathrm{km}$ de moins qu'en réalité. Le Saokoras, $180 \mathrm{~km}$ vers l'aval, apparaît ainsi comme nettement dissocié du Balikh et du Khabûr à la fois. De tels calculs sont généralement peu comparables avec les données de la géographie moderne, mais dans ce cas la différence est trop importante pour qu'elle puisse être attribuée à une erreur d'appréciation.

Plus compliqué est la théorie avancée par Ernst Herzfeld $\left({ }^{13}\right)$. Ce savant supposa que Ptolémée connaissait l'existence du Wadi Tharthar, ce cours d'eau pérenne issu du Jebel Sinjar qui traverse la Mésopotamie en passant près de Hatra, pour se perdre ensuite dans le désert sans atteindre l'un ou l'autre fleuve. Le géographe aurait simplement admis, ou en était informé ainsi, que le Tharthar se jette dans l'Euphrate. À l'appui de sa thèse, Herzfeld voulait utiliser les renseignements des géographes arabes, tels Ibn Sarapion et Yâqût (respectivement du $\mathrm{x}^{\mathrm{e}}$ et du XIII ${ }^{e}$ siècle), qui rattachaient le Tharthar au Hermâs, l'antique Mygdonios et le moderne Jaghjagh, le cours d'eau venant du côté de Nisibe pour rejoindre le Khabûr juste en amont de Hassakeh ${ }^{(14)}$. Selon ces données, une digue au lieu-dit Sukayr al-'Abbâs dirigeait le Jaghjagh dans le lit du Tharthar qui aboutirait au Tigre à Takrit. Ce dernier détail est moins commode pour l'identification avec le Saokoras; Herzfeld cite bien un voyageur anglais qui a vu le Tharthar non loin de l'Euphrate, à la latitude de $34^{\circ}$, mais c'était beaucoup trop à l'est, comme on peut le voir sur une bonne carte, sud pour justifier un rapprochement avec le Saokoras de Ptolémée. L'objection majeure consiste cependant dans une impossibilité physique : entre le Jaghjagh et le Tharthar court la chaîne du Sinjar qui empêche rigoureusement leur réunion.

Les sources du Saokoras chez Ptolémée sont placées cependant au nord du Mont Singaras, évidemment Jebel Sinjar, et au sud du Mont Masios, dans la région donc où prennent naissance les ruisseaux qui descendent du piémont du Tûr 'Abdîn et se réunissent pour former le Khabûr. Plus important encore, les coordonnées de ces sources correspondent à celles de la ville de Nisibe, située en fait tout près de la source principale du Jaghjagh. Comme ces relations ne sont certainement pas fortuites, quelle que soit la précision des données numériques, fautil plus pour admettre que les sources du Saokoras sont les mêmes que celles du moderne Jaghjagh et de l'antique Mygdonios ?

En même temps, Ptolémée place les sources du Chaboras pratiquement à la même latitude, mais plus à l'ouest, coïncidant avec la position du Mont Masios, c'est-à-dire le point considéré comme le plus caractéristique de la chaîne du Tûr 'Abdîn. D'habitude, les Anciens plaçaient les sources du Khabûr à Ras el-'Ain, un peu vers le nord-ouest du point donné par Ptolémée ( $\left.{ }^{15}\right)$. Mais c'est toujours la région d'origine des nombreux cours d'eau qui se réunissent pour former le Khabûr proprement dit, qui recevra bientôt le Jaghjagh pour continuer plein sud vers l'Euphrate.

Le Chaboras et le Saokoras commencent donc chez Ptolémée comme les deux branches du Khabûr, mais chacun se jette séparément dans l'Euphrate, ce qui est bien contraire à la réalité. La difficulté subsiste, la même que pour l'hypothèse d'un simple échange des noms. Non content de compliquer les données en introduisant dans l'équation le Wadi Tharthar qui n'a rien à y faire, Herzfeld continue avec une explication du nom de Saokoras qui serait

(13) Memnon, 1, 1907, pp. 218-6, 219.

(14) Cf. G. Le Strange, The Lands of the Eastern Caliphate, Cambridge, 1930, pp. 87, 98.

(15) Cf. M. TARDIEU, Les paysages reliques. Routes et haltes syriennes d'Isidore à Simplicius, Louvain-Paris, 1990, p. 103. 
identique à celui du Sajûr, attesté déjà dans les annales assyriennes comme Sagur $\left({ }^{16}\right)$. Si la ressemblance phonétique est frappante, il reste que ce dernier cours d'eau rejoint l'Euphrate sur la rive droite et presque $400 \mathrm{~km}$ plus au nord, tout près de la frontière turque! Il faut admettre que c'est un prix élevé à payer pour une simple assonance des noms. Qui plus est, une variante manuscrite chez Ptolémée porte «Askoras»; si elle est rejetée par toutes les éditions, elle me parait pourtant aussi bonne que «Saokoras», sauf précisément qu'elle ne s'accommode pas de la comparaison avec le Sajûr. En tout cas, cette comparaison ne saurait témoigner en faveur de la leçon admise.

Admettons donc que, Saokoras ou Askoras, le nom ne soit pas connu par ailleurs. Pour identifier la rivière elle-même, il sera peut-être utile de revoir quel est chez Ptolémée le contexte géographique. Or, parmi les «villes et villages de la Mésopotamie qui sont sur l'Euphrate», il nomme en succession à partir de Chabora les étapes suivantes : Thelda, Aphphadana, Banabê, Zaitha, Bethauna et Reskipha $\left({ }^{17}\right)$. Le confluent du Saokoras est par ailleurs localisé juste avant la dernière nommée. Le lecteur pressé pourra croire que Chabora corresponde à l'embouchure du Chaboras, mais en fait il n'en est ainsi que dans le texte établi par Karl Müller, qui a harmonisé les données. Le texte reçu accuse une différence de 1 à 2 degrés de longitude, selon le manuscrit, et place ainsi le Chaboras, contre toute attente, en amont de Nicéphorion.

TABLE II

Quelques coordonnées chez Ptolémée

\begin{tabular}{|c|c|c|c|c|}
\hline & Longitude & Latitude & \multicolumn{2}{|c|}{ Comparaison } \\
\hline Zeugma & $72^{\circ}$ & $37^{\circ}$ & & \\
\hline Nicphorion & $73^{\circ} 5^{\prime}$ & $35^{\circ} 20^{\prime}$ & & \\
\hline Chaboras, sources & $74^{\circ}$ & $37^{\circ} 15^{\prime}$ & Rhisina & $73^{\circ} 30^{\prime}: 37^{\circ} 30^{\prime}$ \\
\hline & & & Mt Masios & $74^{\circ}: 37^{\circ} 20^{\prime}$ \\
\hline Saokoras, sources & $75^{\circ}$ & $37^{\circ} 30^{\prime}$ & Nisibe & $75^{\circ} 30^{\prime}: 37^{\circ} 30^{\prime}$ \\
\hline & & & Mt Singaras & $76^{\circ} 40^{\prime}: 36^{\circ} 15^{\prime}$ \\
\hline Chaboras, confl. & $72^{\circ}\left(40^{\prime}\right) *$ & $35^{\circ} 15^{\prime} * *$ & & \\
\hline Chabora & $74^{\circ}$ & $35^{\circ} 10^{\prime}$ & & \\
\hline Bethauna & $76^{\circ}$ & $34^{\circ} 15^{\prime}$ & & \\
\hline Saokoras, confl. & $75^{\circ} 45^{\circ}$ & $33^{\circ} 50^{\prime}$ & & \\
\hline & var. $76^{\circ}$ & var. $34^{\circ}$ & & \\
\hline Reskipha & $76^{\circ}$ & $34^{\circ}$ & Palmyre & $34^{\circ}$ \\
\hline
\end{tabular}

* Müller $74^{\circ}$, var. $73^{\circ}$

** Müller $35^{\circ} 10^{\prime}$

Le nom de Zeitha revient encore deux fois dans notre documentation, notamment chez Ammien Marcellin et Zosime (respectivement sous la forme de Zaitha et Zautha) $\left({ }^{18}\right)$, comme le site du tumulus de l'empereur Gordien, visité par Julien au début de sa malheureuse expédition perse. C'était la première étape après la traversée du Khabûr à son embouchure, située à 60 stades de distance selon Zosime, 20 milles selon Eutrope, soit à 11 ou $29 \mathrm{~km}$. Dans l'un ou l'autre cas, il n'y a pas moyen d'intercaler trois stations intermédiaires entre le Khabûr et Zaitha, comme il serait nécessaire si celle-ci était identique à Zeitha de Ptolémée.

(16) Sur le Sajûr, cf. E. Sachau, Reise in Syrien und Mesopotamien, Leipzig, 1883, p. 152. Sagur: $A R A B$, I, 560, 603, 610 (Salmanasar III, en 856 et 853 av. J.-C.).

(17) Ptol., V, 17, 5.

(18) Амм., XXIII, 5, 7 ; Zosıм., III, 14, 2. 
Après Zeitha, le nom de la station suivante chez Ptolémée est donné comme Bethauna par toutes les éditions, même si la variante manuscrite la plus fréquente est Bedauna, et que l'on trouve aussi les formes Baidauna, Baithautha, et même Methauna. Il est communément accepté, depuis Ritter, que ce nom représente la cité insulaire de 'Ana (*Beth- Ana) qui a capitulé devant Julien lorsqu'il l'a atteinte au bout de deux jours de marche et qu'Ammien appelle Anatha. La ressemblance des noms a été décisive pour cette identification, ainsi que pour le choix de la forme du nom dans le texte établi (la princeps a pourtant Baithautha).

L'hypothèse présente de sérieux inconvénients. D'abord, si Ptolémée nomme trois étapes entre Chabora et Zeitha, il ne connaît aucune entre cette dernière localité et Bethauna (si vera lectio); d'après ses coordonnées, le premier secteur est deux fois plus long que le second. Sur le terrain, cependant, la distance entre le site présumée de Zeitha et celui de 'Ana est cinq fois plus longue que celle entre Zeitha et le Khabûr.

J'emprunte ces observations, parfaitement exactes, à $\mathrm{H}$. Kiepert qui y voit un exemple particulièrement flagrant de la qualité détestable des renseignements de Ptolémée : ils seraient presque inutilisables pour la recherche $\left({ }^{19}\right)$ ! Je pense au contraire que les renseignements de Ptolémée, et en particulier son ordre d'énumération des étapes, sont tout à fait estimables, et que ce sont les chercheurs modernes qui se sont trompés en faisant inconditionnellement confiance à leurs propres rapprochements étymologiques, de préférence aux données premières des textes et du terrain.

Il y a d'ailleurs plus de difficultés que l'on a vues. L'étape suivante après «Bethauna» s'appelle chez Ptolémée Reskipha et coïncide à peu près avec l'embouchure du Saokoras. Si Bethauna est 'Ana, le Saokoras est Wadi Sûr, comme déjà d'Anville a logiquement conclu. Nous connaissons pourtant le pays mieux que lui ne pouvait le connaître : il serait ridicule de maintenir que ce ravin, au mieux un ruisseau temporaire, puisse représenter une rivière qui prend sa naissance à quelque $300 \mathrm{~km}$ de là.

De plus, le nom de Reskipha est parlant : il veut clairement dire, en araméen, «Tête-duRocher» (*rěs-kepha), donc quelque chose comme «Promontoire» ou «La Roche», et devait désigner un endroit caractérisé par un accident notable. Et pourtant, la berge de l'Euphrate ne présente dans le secteur de 'Ana rien de tel: le plateau désertique sur les deux rives se termine bien par un escarpement rocheux qui s'avance parfois jusqu'au bord de l'eau, mais il est plutôt uniforme, sans saillants ou caps qui auraient justifié un nom comme celui de Reskipha. Je peux en témoigner pour avoir participé à une campagne de sauvetage dans cette région de 1978 à 1980 et circulé souvent, en bateau ou sur l'une ou l'autre rive de l'Euphrate, entre ‘Ana et Bijân quelques 25 km vers l'aval. Il n'y a simplement pas dans ces parages de rocher proéminent qui aurait marqué l'embouchure d'un affluent. Les coordonnées de Ptolémée ne correspondant non plus, on s'aperçoit que l'opinion reçue ne repose que sur un rapprochement douteux des noms qui ne se ressemblent pas vraiment.

L'étape de Zeitha, d'ailleurs, ne peut correspondre non plus à la localité du même nom par où est passé l'empereur Julien, pour la bonne raison que celle-ci est à chercher sur l'autre rive du fleuve. Il me paraît sauter aux yeux que l'armée de Julien a suivi la rive droite après avoir traversé près de Circesium, en passant notamment devant les ruines de Doura-Europos. J'ai déjà signalé ma position et je développe ailleurs ma démonstration de ce point qui a curieusement échappé à tous les commentateurs d'Ammien et de Zosime et aux historiens

(19) H. KIEPERT, Formae orbis antiqui, 1910, p. 5. 
de Julien $\left({ }^{20}\right)$. Pour l'argument présent, l'itinéraire de Julien est sans importance : la ville de 'Ana (Anatho d'Isidore) était sur une île et pouvait donc être mentionnée tout aussi bien parmi les localités de la rive droite que celles de la rive gauche, prétendument chez Ptolémée. Bethauna ne peut pas être 'Ana pour plusieurs autres raisons, contre lesquelles la ressemblance assez relative des deux noms ne saurait prévaloir.

On peut se demander, bien entendu, comment Ptolémée aurait-il pu omettre cette importante étape de la route de l'Euphrate, bien attestée sous le même nom au fil des siècles, et notamment à l'époque du Géographe. En effet, aucun nom qu'il cite sur l'une ou l'autre rive du fleuve ne ressemble même de loin à celui de 'Ana. Il en va de même de Doura-Europos, à moins que cette cité se cache sous le nom de Auzara, Audattha ou Addara, localités mentionnées dans cet ordre parmi les villes et villages de l'Arabie Déserte situées sur l'Euphrate ; Dussaud s'est prononcé pour Addara $\left.{ }^{21}\right)$, mais le nom courant de la ville était à l'époque Europos, l'usage de celui de Doura s'imposant seulement au cours du III $^{\mathrm{e}}$ siècle. Il ne semble pas que l'on puisse résoudre cette question avec les données disponibles. Il vaut mieux admettre une omission que d'appliquer au texte reçu des moyens procrustéens.

Pour revenir à Reskipha, il semble que la localisation de ce site, l'association de voisinage avec 'Ana une fois écartée, s'impose d'elle-même. En suivant la rive gauche de l'Euphrate après son confluent avec le Khabûr, on arrive au bout de $83 \mathrm{~km}$ au site de 'Irsî. C'est une falaise qui barre la vallée et s'élève directement au bord de l'eau forçant le chemin à monter sur le plateau. Il semble bien que c'est par là que les troupes de Tukulti-Ninurta II, qui en 885 av. J.-C. suivaient l'Euphrate en direction du nord, ont dû se frayer le chemin avec des pics de fer par la montagne "à la tête de l'Euphrate» (ana riš Puratti) pour passer la nuit dans la ville de Nagiatu, à cinq étapes après Anat et autant avant le Khabûr ${ }^{(22)}$. Le site a été rapidement fouillé et étudié par R. du Mesnil du Buisson qui le nomme Baghûz d'après un «village assez misérable» de la plaine ; il y a trouvé une nécropole de l'Âge du Bronze, mais point de ruines d'une cité ( ${ }^{23}$ ). Avant lui, la falaise a eu droit à l'attention de quelques voyageurs à cause des tours funéraires d'époque romaine qu'elle porte $\left({ }^{24}\right)$. Située juste à la frontière syro-iraquienne, en face d'Abu-Kemâl, la falaise de 'Irsî est actuellement inaccessible.

Mais où donc est passé Saokoras ? Son lit est toujours là, au pied de la falaise. Il s'agit d'un important canal que les sources arabes du Moyen Âge nomment Nahr Dawwarîn et qui s'embranchait sur le Khabûr pour couper la plaine sur $120 \mathrm{~km}$ de long avant de toucher l'Euphrate à 'Irsî. Bien visible sur les photos aériennes qu'a utilisées du Mesnil du Buisson, le lit du canal a été récemment étudié par Monchambert et Margueron $\left({ }^{25}\right)$; il paraît que son tracé remonte au troisième millénaire et qu'il servait principalement à la navigation. Large uniformément de $11 \mathrm{~m}$, il pouvait facilement passer pour une rivière naturelle. Ptolémée l'a rattaché au Jaghjagh, ce qui n'est pas totalement faux : les sources d'un cours d'eau qui contribue à former le Khabûr et l'issue d'un canal qui était branché sur cette rivière ont été dissociées

(20) M. Gawlikowski, «La route de l'Euphrate d'Isidore à Julien», dans : P.-L. Gatier, B. Helly, J.-P. ReyCoquais (éd.), Géographie historique du Proche-Orient, p. 89 sq.

(21) R. Dussaud, Topographie historique de la Syrie antique et médiévale, Paris, 1927, p. 456.

(22) A. K. Grayson, Assyrian Royal Inscriptions, 2, Wiesbaden, 1976, p.102 sq.

(23) R. du Mesnil du Buisson, Baghouz, l'ancienne Corsôté, Leiden, 1948.

(24) G. L. Bell, Amurath to Amurath, New York, 1911, p. 82 et fig. 47 sq. ; A. Musı,, The Middle Euphrates, New York, 1927, p. 172.

(25) J.-Cl. Margueron, "L'aménagement de la région de Mari», dans: Techniques et pratiques hydro-agricoles traditionnelles en domaine irrigué, Paris, 1990, pp. 171-191, avec carte p. 181 ; J.-Y. MonchamBerT, ibid., pp. 93 sqq. 
de celle-ci pour en faire une autre, entièrement indépendante. Si erreur il y a, elle est au moins compréhensible.

On m'objectera ici que l'hypothèse reste en contradiction avec la distance réelle entre le Nahr Dawwarîn et le Khabûr. Mesurée sur le terrain, la piste de la rive gauche égale 83 km (26), alors que la distance calculée d'après les coordonnées est plus longue plus de deux fois depuis Chabora, plus de trois fois depuis la rivière Chaboras. C'est plus qu'une inexactitude. Nous avons vu, cependant, que ces points sont mal localisés chez Ptolémée.

Reprenons donc le calcul pour un secteur plus long de la route de l'Euphrate : de Nicéphorion à Reskipha, soit de l'embouchure du Balikh à celle du Nahr Dawwarîn / Saokoras d'après mon hypothèse. Cette fois, les cordonnées de Ptolémée donnent un rectangle dont la diagonale égale $248 \mathrm{~km}$. L'itinéraire moderne de la rive gauche donne entre ces deux mêmes points la distance de ... $249 \mathrm{~km}$. C'est tout simplement le même itinéraire, parcouru pareillement dans l'Antiquité et au début de ce siècle par les mêmes pistes à dos de cheval et mesuré par les mêmes moyens approximatifs. Et si l'on veut vérifier encore, on trouvera dans l'itinéraire d'Isidore de Charax la distance de 45 schoenes, soit $247 \mathrm{~km}$, entre Nicéphorion et la station de Merrhan qui correspond par son nom et sa place dans le parcours au site de Mari ; celuici est déjà très près d'Abu Kemâl en face de 'Irsî / Reskipha.

Si l'on compare maintenant la liste d'étapes de Ptolémée et celle d'Isidore, assez différentes de prime abord, on y trouvera quand même des correspondances qui peuvent confirmer l'erreur de Ptolémée. L'embouchure du Khabûr coïncide chez Isidore, tout le monde est d'accord, avec la station de Phaliga. C'est la sixième ou septième étape après Nicéphorion, alors que Chabora de Ptolémée n'est que la troisième. Si on accepte, comme on le fait d'habitude, que Thelda et Aphadana de Ptolémée (qui suivent Chabora) correspondent à Thillada Mirrada et Basileia d'Isidore (qui précèdent Phaliga), la transposition devient manifeste. Et puisque Chabora comme nom de lieu n'apparaît que chez Ptolémée, il est permis de penser que ce nom est défiguré $\left({ }^{27}\right)$, surtout lorsque l'on constate que les manuscrits le dissocient de la rivière homonyme.

Celle-ci n'est pas simplement confondue avec le Balikh, parce qu'elle est localisée en amont de la ville de Nicéphorion, elle-même située en réalité en amont du Balikh. La différence n'est pas très grande cette fois, mais la position relative est renversée. De plus, le Géographe attribue à son Chaboras les sources du vrai Khabûr, au pied du Mont Masios. Il y a plus que l'échange des noms, il y a une confusion majeure. Karl Müller n'a fait qu'ajouter à ce malentendu, lorsqu'il s'est cru obligé de corriger la position de l'embouchure de la rivière d'après la localité qui en réalité devait s'appeler autrement et n'avait rien à voir avec le Khabûr ${ }^{28}$. Mais cette position était fausse de toute façon.

Si la recherche moderne accepta unanimement cette localisation et en y ajouta d'autres, également fausses, comme celle de Bethauna-Ana, cela résulte en premier lieu de ce que l'on ne prenait pas au sérieux les données numériques de Ptolémée, c'est-à-dire l'essentiel de sa contribution, sous prétexte qu'elles sont inexactes. On y préférait systématiquement l'approche linguistique, la comparaison des toponymes anciens et modernes, exercée souvent avec une grande liberté. Ainsi, par exemple, le site de Doura-Europos a été baptisé Agalgala (Ptolémée

(26) Ici et partout, je calcule les distances réelles d'après le précieux Handbook of Mesopotamia (Great Britain, Naval Intelligence Division, 1917), vol. III et IV.

(27) Dussaud, op. cit., p. 465, corrige pour cette raison Chabora en Choumbanè, comme chez Isidore.

(28) Muller, GGM, I, p. 1001, $19: 6$ mss. portent $72^{\circ}$ et $15 \mathrm{mss} .72^{\circ} 40^{\prime}$; aucun n'a $74^{\circ}$, adopté pourtant d'après p. $1003,11$. 
donne Balagala), par un rapprochement avec le nom turc Khan Qalesi ( $\left.{ }^{29}\right)$, et celui de Dibsi a été pris pour une déformation de Thapsaque. Dans les deux cas, pourtant, il s'agit de noms récents, ne remontant peut-être pas au-delà du XIX ${ }^{\mathrm{e}}$ siècle $\left({ }^{30}\right)$, la toponymie du Moyen-Euphrate étant en général renouvelée après une longue éclipse de la vie sédentaire.

Je crois donc avoir démontré que le Saokoras de Ptolémée correspond à son embouchure au canal de Nahr Dawwarîn, alors que ses sources sont celles du Mygdonios / Jaghjagh. En même temps, les sources du Khabûr sont définies correctement chez Ptolémée, mais son cours inférieur est fortement déplacé vers l'ouest, au point de recouvrir celui du Balikh. Cette situation rappelle curieusement la perception du pays prêtée à Xénophon. Lui aussi, en 401 av. J.-C., ne connaissait que deux affluents de la rive gauche de l'Euphrate, ce qui répond bien entendu à la réalité géographique, mais il est communément admis parmi ses commentateurs qu'il n'a vu que le Khabûr et le Nahr Dawwarîn.

Rappelons les données du texte ${ }^{\left({ }^{31}\right)}$. L'armée de Cyrus le Jeune dont l'écrivain faisait partie a traversé l'Euphrate à Thapsaque, pour suivre la rive gauche à travers le pays que Xénophon appelle la Syrie. Au bout de 9 jours et 50 parasanges, soit quelque $250 \mathrm{~km}$, les Grecs ont atteint la rivière Araxe. De là, ils continuaient pendant cinq jours et 35 parasanges à travers le désert d'Arabie, une vaste plaine rappelant la mer, ayant rencontré force gibier mais point d'habitants. Après 35 parasanges ou $175 \mathrm{~km}$ environ, ils sont arrivés à la rivière Maskas dont le confluent avec l'Euphrate entourait les ruines d'une importante ville nommée Korsôté ( ${ }^{32}$ ). Celle-ci est réputée avoir occupé le site de Baghûz sous la falaise de 'Irsî. Le Maskas serait donc identique au Nahr Dawwarîn.

À lire la description de Xénophon, on est cependant frappé de voir combien elle rend mal compte de cette situation présumée. Gertrude Bell avait besoin de trois jours pour passer de la bouche du Khabûr à 'Irsî ; le canal était à sec depuis longtemps, "une heure à une demiheure du fleuven, mais toujours bien visible, comme aussi les restes d'ouvrages d'irrigation ( ${ }^{33}$ ). Comparer un tel paysage à une mer sans limites serait de la part de Xénophon tout à fait inadéquat, surtout si le canal restait en eau. Il était d'ailleurs uniformément large de $11 \mathrm{~m}$, d'après les relevés de Monchambert, et non d'un plèthre (100 pieds), comme le Maskas de Xénophon. Cinq jours pour traverser ces quelque $80 \mathrm{~km}$, c'est beaucoup ; ne pas y rencontrer âme qui vive dans ce pays irrigué, mais seulement des onagres et des autruches, c'est improbable.

Le récit de Xénophon a été mal interprété à cause d'une idée reçue qui localise Thapsaque vers le grand coude de l'Euphrate, c'est-à-dire peu en amont du Balikh. S'il faut chercher ensuite deux cours d'eau bien éloignés vers l'aval, ce ne pourrait être en effet que le Khabûr et le canal de Dawwarîn, même si les circonstances de la marche ne correspondent pas à la réalité du pays. Cependant, comme je le démontre ailleurs, Thapsaque devait être identique à Zeugma ( $\left.{ }^{34}\right)$, loin vers le nord, déjà en Turquie actuelle. Du coup, on s'aperçoit que les distances de Xénophon se mettent en place : 50 parasanges de Thapsaque à la rivière Araxe, c'est $260 \mathrm{~km}$ de Zeugma à Raqqa près de la bouche du Balikh; 35 parasanges d'Araxe au Maskas, c'est $180 \mathrm{~km}$ de Raqqa au Khabûr. Le site de Korsôté ne sera autre que celui de

(29) E. Herzfeld, dans F. Sarre, E. Herzfeld, Archäologische Reise im Euphrat- und Tigrisgebiet, II, Berlin, 1920, p. 395.

(30) Pour Dibsi et ses noms antiques, voir R. P. HarPER, "Athis-Neocaesareia-Qasrin-Dibsi Faraj», dans : J.-Cl. Margueron (éd.), Le Moyen-Euphrate, zone de contacts et d'échanges, Strasbourg, 1977, pp. 327-348.

(31) Anabase I, IV, 18 - V, 4.

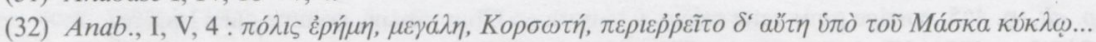

(33) G. L. Bell, Amurath to Amurath, New York, 1911, pp. 78-83.

(34) «Thapsacus et Zeugma», à paraitre. 
Circesium, la dernière place-forte du Bas-Empire sur la route de l'Euphrate, effectivement protégé par le confluent : à lire la description d'Ammien Marcellin (35), on a l'impression d'une paraphrase de Xénophon. Il ne sert à rien de comparer les noms d'Araxe et de 'Irsî, même si l'on écrit al-'Arsi ou 'Arasi pour faciliter la transition ${ }^{\left({ }^{36}\right)}$. La rivière ne porte pas le nom de cette montagne.

Une fois arrivé à cette conclusion, j'ai été bien surpris de la trouver déjà exprimée on ne peut plus clairement en 1913. Le Colonel Arthur Boucher a publié cette année-là un «commentaire historique et militaire», où l'itinéraire de Cyrus et la retraite des Dix-Mille sont reconstitués «d'après les lois de la stratégie, d'une manière rigoureusement conforme aux données de l'Anabase» $\left.{ }^{37}\right)$. Cet ouvrage plein de bon sens et témoignant d'une familiarité avec les auteurs anciens est peu académique dans sa forme ; il a donc été universellement ignoré, il faut craindre sans examen. C'est pourtant Boucher qui avait raison contre tous les professeurs réunis.

Un demi-siècle plus tard, un autre officier, anglais cette fois, amateur de Xénophon, a mis à profit son service de guerre au Proche-Orient pour analyser à son tour l'itinéraire de la marche de Cyrus ( $\left.{ }^{38}\right)$. Il ignorait le travail de Boucher, mais il est arrivé aux mêmes conclusions. Sa notice posthume n'a pas attiré beaucoup d'attention. Cependant, le site de Korsôté a été estimé identique à l'Haradu des Assyriens (prétendument corrompu en Kopjó $\omega \tau \eta \dot{n}$, c'est-à-dire la ruine de Diniyeh récemment fouillée par Chr. Kepinski, alors que le Maskas serait «un canal disparu» $\left({ }^{39}\right)$; cette proposition est irrecevable, car Diniyeh est situé sur la rive droite.

En rendant justice à la perspicacité de ces deux militaires, on gagnera en même temps un renseignement historique intéressant. Au lieu d'une incompréhensible Arabie sur les bancs de l'antique canal du Khabûr, nous voyons ce nom appliqué à la steppe désertique entre cette rivière et le Balikh. Plus au nord, la future Osrhoëne, avec ses «nombreux villages remplis de vin et de blé» est qualifiée de "Syrie» $\left({ }^{40}\right)$; c'est l'opposition entre le pays sédentaire et le domaine des nomades, «the Desert and the Sown».

Il est certain que la distinction entre ces deux régions n'était pas fondée, pour les mercenaires grecs et leurs informateurs, sur des considérations ethniques, surtout qu'ils n'ont rencontré personne dans cette "Arabie». Ce nom n'est pas un malentendu, comme l'a cru un auteur récent ${ }^{\left({ }^{4}\right)}$. Si le pays s'appelait ainsi, c'est qu'il était certainement parcouru par des nomades, même s'ils n'ont pas jugé utile de se montrer à cette occasion. Ils ont toujours été appelés 'Arab par leurs voisins sédentaires, ceci au moins depuis les annales assyriennes du $\mathrm{IX}^{\mathrm{e}}$ siècle, et le même nom leur sera encore appliqué par les inscriptions de Hatra du II $^{\mathrm{e}}$-III ${ }^{\mathrm{e}}$ siècle ap. J.-C., toujours dans la même région.

Michel Gawlikowski (Varsovie, Princeton)

(35) Aмmien, 23, 5, 5: Cercusium ... cuius moenia Abora et Euphrates ambiunt flumina velut spatium insulare fingentes... ; cf. dans la même veine Zosime, 3, 12, 3 et Malalas (FHG, IV, p. 4, s.v. Magmus).

(36) Ainsi A. Musil, The Middle Euphrates, New York, 1927, p. 221.

(37) A. Boucher, L'Anabase de Xénophon, Paris, 1913.

(38) W. J. FARrell, "A Revised Itinerary of the Route Followed by Cyrus the Younger through Syria, $401 \mathrm{BC}$ ", $J H S, 81,1961$, pp. 153 sqq .

(39) R. D. Barnett, JHS, 83, 1963, p. 3 sq. Sur Khirbet ed-Diniye, cf. maintenant C. KePINSK1-LeCONTE (dir.), Haradum I. Une ville nouvelle sur le Moyen Euphrate, Paris, 1992.

(40) Cf. Th. Nöldeke, «Assyrios, Syrios, Syros», Hermes, 5, 1871, pp. 443 sqq. et récemment R. N. Frye, «Assyria and Syria : Synonyms», JNES, 51, 1992, pp. 281-285.

(41) F. M. Donner, «Xenophon's Arabia», Iraq, 48, 1986, pp. 1-14. 